\title{
Linking programmes and systems: lessons from the GAVI Health Systems Strengthening window
}

\author{
B. Galichet', L. Goeman ${ }^{2}$, P. S. Hill ${ }^{3}$, M. S. Essengue ${ }^{4}$, N. Hammami ${ }^{5}$, D. Porignon ${ }^{6}$, P. Kadama ${ }^{6}$ and \\ W. Van Lerberghe ${ }^{6}$ \\ 1 Department of Country Focus, World Health Organization, Geneva, Switzerland \\ 2 Project SISKES PLUS, German Technical Cooperation, Kupang, Indonesia \\ 3 University of Queensland, School of Population Health, Brisbane, Australia \\ 4 GAVI Alliance, Geneva, Switzerland \\ 5 Child Welfare Scheme, Pokhara, Nepal \\ 6 Department of Health System Governance and Service Delivery, World Health Organization, Geneva, Switzerland
}

Summary

OBJEctives To analyse the first four rounds of country applications to the GAVI Alliance Health Systems Strengthening (GAVI-HSS) funding window; to provide valuable insight into how countries prioritize, articulate and propose solutions for health system constraints through the GAVI-HSS application process and to examine the extent to which this process embodies alignment and harmonization, Principles of the Paris Declaration.

METHODs The study applied multiple criteria to analyse 48 funding applications from 40 countries, submitted in the first four rounds, focusing on the country analysis of health systems constraints, coordination mechanisms, alignment with national and sector planning processes, inclusiveness of the planning processes and stakeholder engagement.

RESUlTS The applications showed diversity in the health systems constraints identified and the activities proposed. Requirements of GAVI for sector oversight and coordination, and the management of the application process through the Ministry of Health's Planning Department, resulted in strong alignment with government policy and planning processes and good levels of stakeholder inclusion and local technical support (TS).

CONCLUSION Health Systems Strengthening initiatives for global health partnerships (GHPs) can provide a link between the programmatic and the systemic, influencing policy alignment and harmonization of processes. The applications strengthened in-country coordination and planning, with countries using existing health sector assessments to identify system constraints, and to propose. Analyses also produced evidence of broad stakeholder inclusiveness, a good degree of proposal alignment with national health plans and policy documents, and engagement of a largely domestic TS network. While the effectiveness of the proposed interventions cannot be determined from this data, the findings provide support for the GAVIHSS initiative as implementation continues and evaluation begins.

keywords GAVI-HSS, Health Systems Strengthening, alignment, Ownership, global health partnership

\section{Introduction}

In 2006, the GAVI Alliance (GAVI) opened a Health Systems Strengthening (HSS) funding window to 72 low income countries. The initial US\$500 million was complemented by an additional US\$300 million announced in 2008. Forty eligible countries submitted 49 applications in the first four rounds of funding. This study is an exploratory study of the GAVI-HSS application process, based primarily on documentary evidence. It provides insight into how developing countries articulate their intentions in responding to health systems constraints, though at this stage, it is not possible to draw any conclusions regarding the effectiveness of the interventions proposed.

The GAVI-HSS process is both predictable and noncompetitive. Countries are allocated a figure already calculated on Gross National Income (GNI) and annual births. Ministries of Health are invited to use currently available sectoral reviews to identify health systems constraints and to plan responses that will strengthen the health system, and in doing so, improve coverage of immunization and maternal and child health care. The funding is to be considered as additional and complementary to existing sources. Accountability is reflected in the 
B. Galichet et al. Linking programmes and systems

commitment of GAVI-HSS to demonstrable outcomes and the selection of measurable monitoring and evaluation indicators (GAVI Alliance 2006).

GAVI-HSS requires that the planning process be located in the Planning Departments of Ministries of Health, under the supervision of Health Sector Coordinating Committees (HSCC). This highlights the sectoral orientation of this initiative, moving responsibility away from immunization programme management. The documentation of broad stakeholder participation throughout the application development process is intended to further reinforce harmonization, and avoid further fragmentation. The process avoids prescriptive blueprints, and is designed to be both inclusive and collaborative, producing proposals that are aligned with the countries' policies, planning processes and budgetary cycles. This is consistent with the commitments of the Paris Declarations on Aid Effectiveness (OECD 2005). These objectives reflect recognition that even specific targets - such as the health-related Millennium Development Goals (MDGs) - are dependent on change at the systems level (Travis et al. 2004).

Applications are evaluated by an Independent Review Committee (IRC), appointed on the basis of technical expertise and familiarity with country contexts. They are responsible for the evaluation of the proposals' content and process, and for ensuring their overall coherence. The IRC uses the GAVI-HSS guidelines but has autonomy in decision making, and is authorized to approve applications, if necessary after clarification or with conditions. Criteria for approval include clear linkage of the proposal to national health plans (NHPs) and immunization outcomes, endorsement by Ministries of Health and Finance and relevant development partners, valid indicators and appropriate monitoring and evaluation mechanisms (GAVI Alliance 2006). The assessment process is interactive, and applications which do not meet the guidelines are to be referred for resubmission, rather than rejected outright. Approval from the GAVI board is required prior to notification of acceptance.

\section{Methods}

This analysis of the GAVI-HSS application process draws on the content of all 48 application forms submitted by GAVI-eligible countries between October 2006 and October 2007 (rounds 1-4), building on a preliminary study of the first three rounds (Hill et al. 2007). Eight proposals resubmitted in subsequent rounds have been included in this analysis, given the extensive changes in content required. One additional application submitted as a twopart proposal had been excluded. At the time of this study, 21 of the 48 applications had been fully approved or approved subject to clarification. Fourteen had been conditionally approved by the IRC, and a further 13 applications were recommended for resubmission. An overall analysis of the IRC reports showed that the complex of reasons for resubmission tended to cluster in four principal areas: (i) content and coherence of planning; (ii) implementation, management, and monitoring and evaluation; (iii) budget allocations; and (iv) inclusiveness and complementariness.

A subset of 29 applications, submitted in rounds 3 and 4, using a revised and more detailed application form, was used for the analysis of specific aspects of the proposal development process.

\section{Analysis of constraints and proposed activities}

The research team analysed health system constraints identified by in-country health planners and linked to activities proposed in the applications. The classification for analysis of constraints and activities was adapted from WHO's Everybody's business: strengthening health systems to improve health outcomes (WHO 2007). Inter-rater reliability of allocation to these categories was high, with a kappa score (Jekel et al. 2001) of 0.88. Constraints and activities were classified by level as either operational or systemic. Operational constraints were considered to be primarily at the district level or below and addressed with funding or resources. Systemic constraints were those that required more comprehensive systems changes and could not be addressed with funds or resources alone.

\section{Alignment}

Analysis of the applications for degree of alignment was undertaken independently by two analysts using the same criteria. Proposals were awarded a score from 1-5 by each analyst, with a score of 1 being a proposal fully aligned with the NHP content and budget cycle, and 5, a proposal in independent project format. Scoring was based on a combination of three objectively verifiable core criteria with four more subjective complementary criteria (Table 1). Agreement between analysts occurred in the assessment of 37 of the 48 applications, with a Kappa score of 0.69 suggesting a good level of agreement (Table 2) (Jekel et al. 2001). After discussion and negotiation between the two analysts, a final consensus was secured and one agreed score allocated to each proposal.

\section{Coordination, inclusiveness and stakeholder involvement}

Analyses of coordination committee structures, inclusiveness and levels of technical support (TS) were undertaken 
B. Galichet et al. Linking programmes and systems

Table I Criteria for assessment of degree of alignment

Assessment criteria

\begin{tabular}{|c|c|c|}
\hline Core criteria & Complementary criteria & Degree of alignment \\
\hline $\begin{array}{l}\text { 1. Demonstrated alignment with the } \\
\text { budget cycle as indicated in the NHP, } \\
\text { the Comprehensive Multi-Year Plan } \\
\text { (cMYP) for immunization, or the } \\
\text { Mid-Term Expenditure Framework } \\
\text { (MTEF) } \\
\text { 2. Comparison of content of health } \\
\text { systems issues identified the } \\
\text { application and the NHP } \\
\text { 3. Implementation through existing } \\
\text { structures and mechanisms }\end{array}$ & $\begin{array}{l}\text { 1. A broad health systems focus } \\
\text { 2. Planning coherence (consistency } \\
\text { between activities and constraints) } \\
\text { 3. Complementarity with other } \\
\text { funding sources } \\
\text { 4. Evidence of supportive interactions } \\
\text { with the NHP and other health } \\
\text { sector documentation }\end{array}$ & $\begin{array}{l}\text { Fully aligned: all three objectively } \\
\text { verifiable core criteria showed evidence } \\
\text { of alignment, this was confirmed } \\
\text { through complementary criteria } \\
\text { Mainly aligned: evidence for one of the } \\
\text { three core criteria is missing, with } \\
\text { complementary criteria either confirming } \\
\text { perceptions of alignment, or neutral } \\
\text { Partly aligned: evidence for two core } \\
\text { criteria are missing, or <50\% of areas of } \\
\text { support correspond with the NHP (e.g. } \\
\text { five areas of support in Health Systems } \\
\text { Strengthening, but only two in the } \\
\text { NHP), with complementary criteria } \\
\text { either confirming perceptions of } \\
\text { alignment or neutral } \\
\text { Poorly aligned: evidence for two core } \\
\text { criteria are missing, with complementary } \\
\text { criteria not corroborating perceptions of } \\
\text { alignment, or providing evidence } \\
\text { questioning alignment } \\
\text { Unaligned: three core criteria are missing } \\
\text { or the proposal is presented in } \\
\text { independent project format with a } \\
\text { programmatic rather than a system focus }\end{array}$ \\
\hline
\end{tabular}

$\mathrm{NIH}$, national health plans.

on the subset of 29 applications received in rounds 3 and 4, as application forms from previous rounds did not elicit information suitable for this purpose.

Assessment of the inclusiveness of the application process was based on four types of documentary criteria: (i) evidence of a Health Systems Coordination Committee meeting at least twice a year; (ii) the participation of at least four international stakeholders in the application process; (iii) presence of one or more of: the private sector, civil society, independent health professionals, academics (WHO 2000); and (iv) documentary evidence of stakeholder attendance at preparatory meetings. Each factor scored a single point. Applications that satisfied all four criteria (4 points) were considered highly inclusive, three criteria partly, two criteria poorly inclusive, and those that met none or only one criterion ( $0-1$ point) were considered non-inclusive. Assessment was undertaken by two independent analysts, who found agreement on 26 of the 29 allocations, with a Kappa score of 0.84 (Table 2).

Stakeholder participation was examined in further detail: enumerated by agency, differentiating between stakeholders within the government, external stakeholders in-country, and international external stakeholders. Stakeholders were
Table 2 Initial assessment and final consensus on degree of alignment and degree of inclusiveness of the proposal development process

\begin{tabular}{|c|c|c|c|}
\hline \multirow[b]{2}{*}{ Score } & \multicolumn{2}{|c|}{ Initial analysis } & \multirow{2}{*}{$\frac{\text { Final consensus }}{\text { Analysts } 1 \text { and } 2}$} \\
\hline & Analyst 1 & Analyst 2 & \\
\hline \multicolumn{4}{|l|}{ Alignment $(n=48)$} \\
\hline Fully & 13 & 16 & 14 \\
\hline Mainly & 17 & 16 & 18 \\
\hline Partially & 7 & 6 & 6 \\
\hline Poorly & 7 & 6 & 6 \\
\hline Unaligned & 4 & 4 & 4 \\
\hline \multicolumn{4}{|c|}{ Inclusiveness $(n=29)$} \\
\hline Not inclusive & 2 & 2 & 2 \\
\hline Poorly inclusive & 3 & 4 & 3 \\
\hline Partly inclusive & 11 & 12 & 11 \\
\hline Inclusive & 13 & 11 & 13 \\
\hline
\end{tabular}

defined as agencies identified within the application as participating in its drafting, review or endorsement.

Technical support represented a sub-set of stakeholder participation and was identified from the responses to the question 'who led the drafting of the application and was any technical assistance provided?' and in the 'Roles and responsibilities of key partners' table in the application 
forms. TS providers were considered as those whose role was to provide technical assistance, support, expertise, input or advice.

The study used the World Bank (WB) Low Income Countries Under Stress (LICUS) criteria for the fiscal year 2006 to identify applications coming from severe, core or marginal LICUS states (Independent Evaluation Group 2007). The LICUS classification reflects level of income, and an assessment of national policies, institutions and public sector management. It was selected on the basis of its comprehensiveness, wide acceptance and the direct correspondence of its time frame (2006) to the application process (Ikpe 2007). Eighteen applications were received from LICUS states.

The median, range and standard deviations were calculated using Epi-Info, version 3.3.4 (Centers for Disease Control and Prevention Atlanta, Division of Integrated Surveillance Systems and Services, Altlanta, GA, USA), and used the Mann-Whitney two-sample statistical test for statistical comparison of the median. Data were tabulated using Microsoft Excel 2007 spreadsheet functions with selective filters to enable analyses.

\section{Limitations of the study}

This article relies on an analysis of data submitted as part of a funding application, rather than a research process. As such, we are unable to assess whether the constraints identified were the most important, or whether the proposed interventions will effectively address these constraints. Despite concerns raised regarding the quality of the applications (Naimoli 2009), the iterative nature of the process, and interaction with the IRC, substantially improved reviewed applications, and with increasing experience, the overall quality of applications has improved in the fourth round. Although the value of secondary data analysis is increasingly recognized (Shi 2008), country attempts to satisfy donor requirements may have affected the data through reporting bias. Anecdotal, extra-documentary evidence suggests that observed practice may be at variance with the evidence reported in the application. To minimize the impact of these limitations, we have triangulated our assessments with the reports and recommendations of the health systems experts in the IRC, and corroborated with WHO regional and country offices involved in the application development.

\section{Results}

\section{Identified health systems constraints}

Figure 1 shows the distribution of categories of constraints identified by country application, noting approval status and
LICUS status. Of the 462 barriers to improved immunization coverage that were linked to activities in the 48 applications, $258(55.8 \%)$ were at the systemic level, with $204(44.2 \%)$ at the operational level. In more than $93 \%$ of interventions, linkage to immunization was through broader health systems, rather than through the immunization programme. Some countries identified constraints across a wide spectrum of categories, while others focussed on solutions for a more limited set of constraints. At district level, constraints related to service delivery were the most commonly identified, as were leadership and governance constraints at the systemic level. Weak governance and leadership was more consistently identified as a constraint in LICUS states. Detailed analyses of how these constraints were addressed are described elsewhere (Goeman et al. in press).

\section{Alignment with national and sectoral planning processes}

Alignment with in-country planning processes is a central requirement of the GAVI-HSS application development process. The identification of health sector barriers to improved immunization coverage was undertaken by analysing and summarizing recent sector reviews, and assessing priorities not met by other sources, but that could be addressed through GAVI-HSS funds. Policy alignment was confirmed by evidence that the application was consistent with the contents of the NHP, and aligned with the budgetary cycle. The latter condition restricted funding applications to the period remaining in the current cycle.

Of the 48 applications examined for evidence of alignment, $73 \%$ included a NHP covering the period of their proposed funding, or its equivalent. Four submitted planning documentation covering a shorter period than their proposal, but aligned with the Comprehensive Multi-Year Plans (cMYP) for immunization or the Mid-Term Expenditure Framework (MTEF). Nine applications (19\%) were submitted without any planning or strategic documentation and were referred for resubmission. Two countries capitalized on the catalytic nature of GAVI-HSS, using it as an opportunity to finalize work on their NHPs, as well as a stimulus for planning at lower levels.

Analysis showed alignment of the 48 applications to be surprisingly good: $32(67 \%)$ of the applications were graded as either fully aligned $(14,29 \%)$ or mainly aligned $(18$, $38 \%$ ) with the NHP, with a further six applications (13\%) considered partially aligned. Six applications (13\%) were considered poorly aligned, and four $(8 \%)$ were presented in an independent project format, and referred for resubmission. Higher levels of alignment correlated positively with approval, with 26 of 35 approved or conditionally approved proposals $(74 \%)$ considered 'fully' or 'mainly' aligned compared with 6 of the 13 proposals recommended for 
B. Galichet et al. Linking programmes and systems

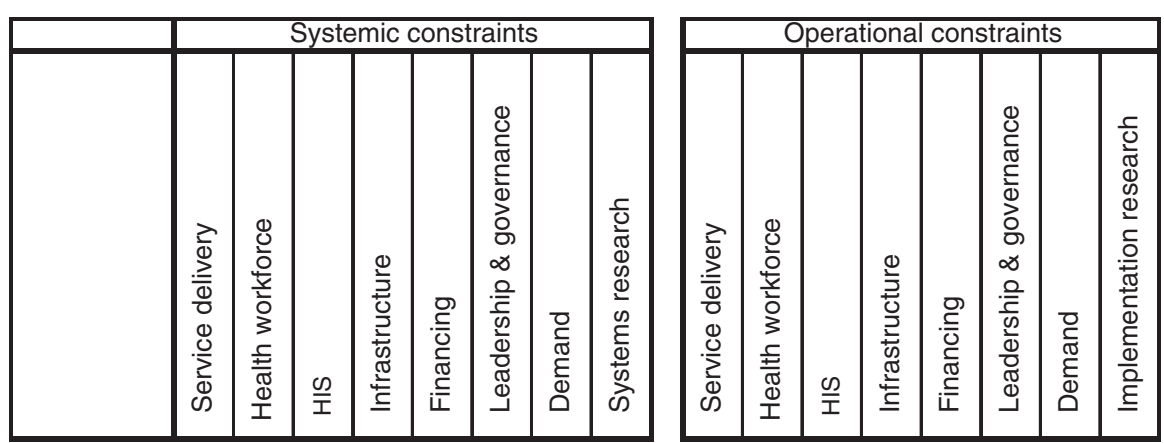
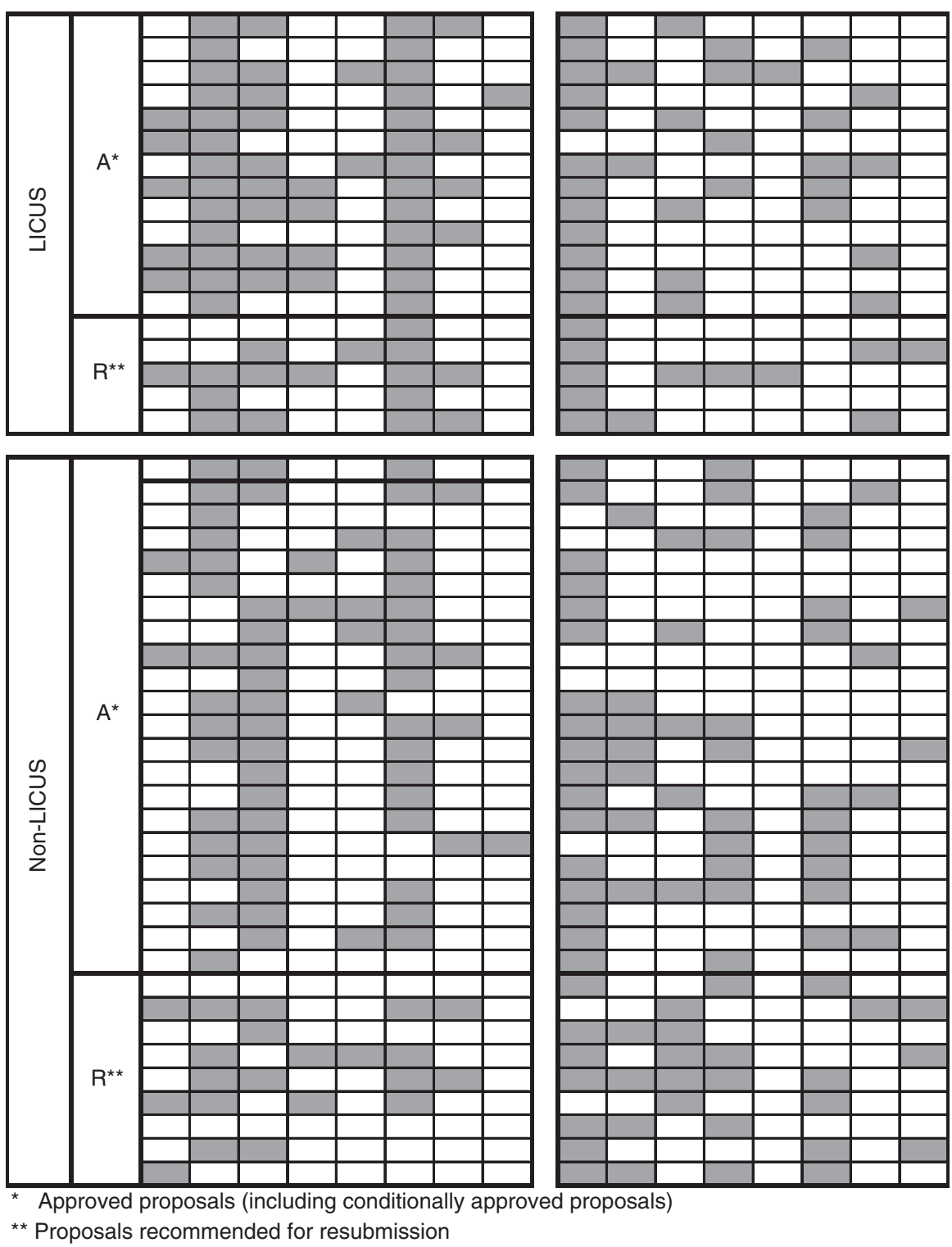

Figure I Distribution of constraints linked to activities, by category, country and Low Income Countries Under Stress status. 
resubmission $(46 \%)(P<0.01)$ (Table 3$)$. The eight original applications that have been revised and resubmitted showed marked improvement in alignment.

\section{Coordination: roles, positioning and function}

The GAVI-HSS guidelines require a functioning national health sector coordination committee, to provide oversight for the application process, implementation, monitoring and evaluation of the proposed activities, and to ensure inclusiveness in planning and implementation. In a number of countries, this has been met with some resistance from the immunization community, but in most cases, such disputes were resolved.

Of the 29 applications providing detail on coordination functions, $20(69 \%)$ described existing HSCC whose role also included the development of the NHP (20 committees), sector coordination (19), HSS (17) or ensuring policy alignment

(16). Most significantly, in nine cases - six of them LICUS states - engaging in the GAVI-HSS application development process was the catalyst for creating coordinating structures.

As part of its strategy to ensure that the GAVI-HSS application process was located within the health system as a whole, the guidelines recommended the Planning Department of the Ministry of Health $(\mathrm{MOH})$ as the appropriate place for the development of the application. Of the 29 applications, 26 located responsibility for planning within the Planning Department, with 24 applications specifying the involvement of Planning Department personnel in the drafting process. Where planning was the responsibility of another department, the IRC sought clarification, to ensure proposal planning was integrated into central $\mathrm{MOH}$ processes. Applications submitted from immunization programmes or departments were recommended for resubmission.

\section{Inclusiveness of the application process}

The assessment of the inclusiveness of the application process resulted in fairly high scores (mean score 3.24 out of 4), with only 3 of 29 assessed applications considered poorly inclusive (score $=2$ ) and two not inclusive ( score $=0$ or 1$)$. Higher levels of inclusiveness correlated positively with approval $(P<0.05)$, with a mean score of 3.5 compared to 2.3 for applications referred for resubmission (Table 4). There was strong correlation between the evaluation of inclusiveness by this study, and the assessment of the proposals by the IRC. This pattern of greater inclusiveness of stakeholders in approved applications was consistent across the three stages of the application process: drafting, review and endorsement although more pronounced during the initial stages.
Table 3 Alignment and approval status of applications

\begin{tabular}{lll}
\hline Alignment $(n=48)$ & $\begin{array}{l}\text { Approved } \\
(n=35)\end{array}$ & $\begin{array}{l}\text { Resubmission } \\
(n=13)\end{array}$ \\
\hline Aligned & 13 & 1 \\
Mainly aligned & 13 & 5 \\
Partly aligned & 5 & 1 \\
Poorly aligned & 4 & 2 \\
Independent project format & 0 & 4 \\
\hline
\end{tabular}

Table 4 Inclusiveness and approval status of applications

\begin{tabular}{lll}
\hline $\begin{array}{l}\text { Inclusiveness } \\
(n=29)\end{array}$ & $\begin{array}{l}\text { Approved } \\
(n=23)\end{array}$ & $\begin{array}{l}\text { Resubmission } \\
(n=6)\end{array}$ \\
\hline Inclusive & 12 & 1 \\
Partly inclusive & 9 & 2 \\
Poorly inclusive & 2 & 1 \\
Not inclusive & 0 & 2 \\
\hline
\end{tabular}

The range of participating stakeholders was broad. Beyond the Health Sector Coordination Committee itself, combinations of eight stakeholder categories were involved in the drafting and review of the applications: the $\mathrm{MOH}$, Ministry of Finance, other government ministries or agencies, bilateral donors, multilateral agencies, health professionals (public or private), domestic universities, civil society and non-government organizations (NGOs).

Stakeholder participation in the drafting phase was dominated by the provision of TS, concentrating local $\mathrm{MOH}$ and multilateral resources to develop the initial draft application. Review broadened the scope of participation, with the Ministry of Finance (16 applications) or other ministries involved (8), and greater engagement of bilateral agencies (18), other multilaterals (15) and local (18) as well as international NGOs and civil society organizations (15). The review processes in particular, provided greater diversity for engagement, with 28 applications reporting review workshops, and 20 citing external stakeholder feedback provided through email contact. A smaller number (9) reported structured peer review processes, with four taking their proposals for field review.

\section{Technical support}

All proposals listed more than one TS provider. The drafting of the applications engaged local TS, with the most common configuration $22 \%$ or $76 \%$ of applications) involving a Technical Working Group with representatives from the $\mathrm{MOH}$, local consultants (most often organized through WHO or GAVI) and other stakeholders. The $\mathrm{MOH}$ was represented in all 29 
B. Galichet et al. Linking programmes and systems

processes, and its Planning Department specifically mentioned in 26 (90\%). Interestingly, Expanded Programme of Immunization (EPI) provided input in only 10 $(35 \%)$ cases, consistent with the broad health systems, rather than EPI focus. The WHO country offices provided TS in 28 applications, and UNICEF country offices in 20 applications.

Technical support from other in-country agencies varied. Fourteen proposals reported civil society and academia, international NGOs and health professionals as having provided TS. The local offices of bilateral agencies provided support in 13 processes, the WB in nine cases and the UNFPA country office in eight cases. Nine applications were assisted through local NGOs, and a further nine through the local offices of international NGOs. The private sector was not reported as playing a role in TS. TS from sources outside the country played a complementary role: WHO regional offices and headquarters participated in 14 applications, and a UNICEF regional office in one application.

A median of six TS providers were involved in the development process for applications. This was positively linked to approval, with approved applications supported by a median of seven TS providers, compared to four in proposals recommended for resubmission $(P<0.05)$.

The role of contracted local and international consultants is of interest. Although GAVI offers applicants a TS grant of US\$ 50000 to assist the application process, recruitment of international consultants was limited. Eleven local academic or independent consultants were engaged, frequently with WHO support, in the drafting process. Nine international consultants were engaged by GAVI (four consultants) or GAVI partners. Five international consultants not related to GAVI provided technical assistance (four were organized by USAID).

\section{Discussion}

The experience of GAVI-HSS provides support for the feasibility of developing a broader HSS approach while starting from (and incorporating), programmatic concerns. It demonstrates the capacity of even LICUS states to develop locally grounded strategic plans when they are given freedom to apply their own judgement without prescriptive guidelines. At the same time, it shows that the use of limited funds can be used to promote policy alignment and harmonization. Such a model may inform other global initiatives that increasingly recognize the need for synergies between systemic capacity and programmatic effectiveness (Brugha et al. 2002; Travis et al. 2004; Lu et al. 2006). In this context, three key lessons have been learnt:
- HSS initiatives can serve to link the programmatic to the systemic

- For GAVI, whose primary mandate lies with immunization, the goal of improved immunization coverage has provided a useful rationale for examining broader health systems constraints, aligning these proposals with broader HSS perspectives. The high proportion of activities that have been proposed with broad health systems, rather than narrow programmatic linkages, suggests that programme specificity has been transcended, and that the GAVI-HSS process has been instrumental in achieving this.

- Providing freedom for planning promotes contextspecific outcomes

- GAVI-HSS has avoided problem specificity by giving a 'free hand' to countries to identify health systems constraints and develop locally appropriate responses, without applying prescriptive blueprints itself. With Ministries encouraged to base their design on analyses that they have selected and endorsed, a technical space has been opened, unconstrained by prescribed disease, programme or systems paradigms, where understanding the local is given priority. The broad diversity of constraints and subsequent intervention activities proposed is a product of the open and flexible format of the GAVI-HSS window. While TS was used, the pattern is towards reliance on local or regional sources, reinforcing the development of coherent, contextualized proposals.

- At the same time, the process has not been without significant transaction costs: it has required structural change, demanding analytic exercises, the coordination of multiple stakeholders and linkage to other planning processes. It has, however, enabled local planners in these low income countries to demonstrate their competence in analysis and planning, and their capacity to engage in developing initiatives addressing health systems (Reich et al. 2008). This is not to say that certain commonalities have not emerged, reflecting shared health systems problems and priorities. Health systems constraints have been mostly addressed in operational responses, with a focus on the gaps in health services supplies, and training and financial incentives for the workforce (Hill et al. 2007; Naimoli 2009). In terms of sustainability, this has implications that will require careful consideration; but the dialogue established with governments through this process should provide a good basis.

- Country-led proposal development can contribute to alignment and harmonization

- The GAVI-HSS process has produced a structural space within the $\mathrm{MOH}$ that promotes policy 
B. Galichet et al. Linking programmes and systems

alignment. The application process has created or reinforced sector coordination structures, integration with central $\mathrm{MOH}$ planning functions and linkage to other sector planning or review processes. In nine cases, the GAVI-HSS process has been a catalyst to establishing this national coordination capacity. In a similar way, by requesting the use of available sector reviews for health system problem diagnosis and the NHP as a referent for these applications, the GAVIHSS process has linked the proposals to a local history of analysis and planning, and alignment with national budgetary cycles. The process has also created a political space for policy alignment, building strong, predominately local coalitions within the $\mathrm{MOH}$ and with key stakeholders (Naimoli 2009) - although these would appear to be largely limited to the government and the donor community, based on reported composition of HSCCs.

\section{Conclusion}

The GAVI-HSS process has shown that it is possible to approach health systems issues with a less directive approach, and greater reliance on the planning capacity of ministries (even in LICUS classified countries), and their local networks of support. Now the challenge for the beneficiary governments and their partners is to ensure the realization of the projected health systems interventions. Only through the evaluation of the outcomes of these interventions will the effectiveness of the process become evident.

\section{References}

Brugha R, Starling M \& Walt G (2002) GAVI, the first steps: lessons for the Global Fund. Lancet 359, 435-438.

GAVI Alliance (2006) Health System Strengthening (HSS). GAVI Alliance, Geneva. http://www.gavialliance.org/vision/policies/ hss/index.php (retrieved February 2008).

Goeman L, Galichet B, Porignon D et al. (in press) Trade-offs: interventions and funding allocation in the first four rounds of the GAVI Health Systems Strengthening applications. Health Policy and Planning.

Hill PS, Hammami N, Essengue M-S, Nchinda T \& Van Lerberghe W (2007) Key Features of the 2006-2007 Proposals: A Report to the GAVI-HSS Task Team. Preliminary Findings from the First 31 Proposals. World Health Organization, Geneva.

Ikpe E (2007) Challenging the discourse on Fragile States. Conflict, Security and Development 77, 84-124.

Independent Evaluation Group (2007) Appendix B. Engaging with Fragile States. World Bank, New York. http://www.worldbank.org/ieg/licus/docs/appendix_b.pdf (retrieved February 2008).

Jekel JF, Katz DL \& Elmore JG (2001) Epidemiology, Biostatistics and Preventive Medicine, 2nd edn. Saunders, Philadelphia, $114 \mathrm{p}$.

Lu C, Michaud CM, Khan K \& Murray CJL (2006) Absorptive capacity and disbursements by the Global Fund to Fight AIDS, Tuberculosis and Malaria: analysis of grant implementation. Lancet 368, 483-488.

Naimoli JF (2009) Global health partnerships in practice: taking stock of the GAVI Alliance's new investment in health systems strengthening. International Journal of Health Planning and Management 24, 3-24.

OECD (2005) Paris Declaration on Aid Effectiveness. OECD, Paris. http://www1.worldbank.org/harmonization/Paris/FI NALPARISDECLARATION.pdf (retrieved February 2008).

Reich MR, Takemi K, Roberts MJ \& Hsiao WC (2008) Global action on health systems: a proposal for the Toyako G8 Summit. Lancet 371, 865-869.

Shi L (2008) Health Services Research Methods, 2nd edn. International Thompson Publishing, Albany NY, 129p.

Travis P, Bennett S, Haines A et al. (2004) Overcoming healthsystems constraints to achieve the Millennium Development Goals. Lancet 364, 900-906.

World Health Organization (2000) Towards Unity for Health: Challenges, and Opportunities for Partnerships in Health Development. WHO, Geneva.

World Health Organization (2007) Everybody's Business: Strengthening Health Systems to Improve Health Outcomes: WHO's Framework for Action. WHO, Geneva. http:// www.who.int/healthsystems/strategy/everybodys_business.pdf (retrieved February 2008).

Corresponding Author Benedicte Galichet, Department of Country Focus, World Health Organization, Via Appia, 1211 Geneva 27, Switzerland. E-mail: bene_galichet@hotmail.com 